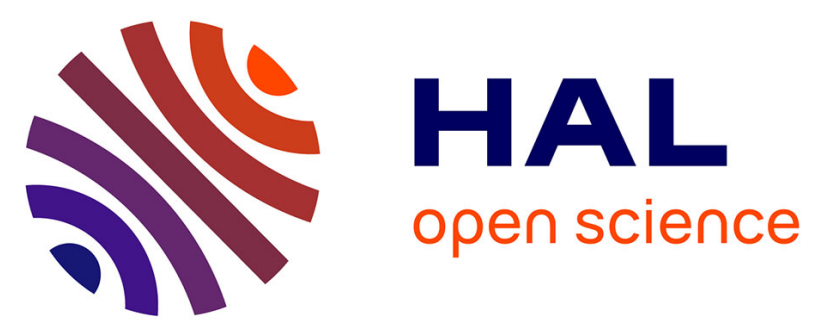

\title{
Novel insights into the regulation of the antioxidant response element mediated gene expression by electrophiles: induction of the transcriptional repressor BACH1 by NRF2
}

\author{
Henna-Kaisa Jyrkkänen, Suvi M Kuosmanen, Merja Heinäniemi, Heidi \\ Laitinen, Emilia Kansanen, Eero Mella-Aho, Hanna Leinonen, Seppo \\ Ylä-Herttuala, Anna-Liisa Levonen
}

\section{To cite this version:}

Henna-Kaisa Jyrkkänen, Suvi M Kuosmanen, Merja Heinäniemi, Heidi Laitinen, Emilia Kansanen, et al. Novel insights into the regulation of the antioxidant response element mediated gene expression by electrophiles: induction of the transcriptional repressor BACH1 by NRF2. Biochemical Journal, 2011, 440 (2), pp.167-174. 10.1042/BJ20110526 . hal-00642837

\section{HAL Id: hal-00642837 https://hal.science/hal-00642837}

Submitted on 19 Nov 2011

HAL is a multi-disciplinary open access archive for the deposit and dissemination of scientific research documents, whether they are published or not. The documents may come from teaching and research institutions in France or abroad, or from public or private research centers.
L'archive ouverte pluridisciplinaire HAL, est destinée au dépôt et à la diffusion de documents scientifiques de niveau recherche, publiés ou non, émanant des établissements d'enseignement et de recherche français ou étrangers, des laboratoires publics ou privés. 


\title{
NOVEL INSIGHTS INTO THE REGULATION OF THE ANTIOXIDANT RESPONSE ELEMENT MEDIATED GENE EXPRESSION BY ELECTROPHILES: INDUCTION OF THE TRANSCRIPTIONAL REPRESSOR BACH1 BY NRF2
}

\author{
Henna-Kaisa Jyrkkänen ${ }^{1}$, Suvi Kuosmanen ${ }^{2}$, Merja Heinäniemi ${ }^{3}$, Heidi Laitinen ${ }^{1}$, Emilia Kansanen ${ }^{1}$, \\ Eero Mella-Aho ${ }^{1}$, Hanna Leinonen ${ }^{1}$, Seppo Ylä-Herttuala ${ }^{1}$, Anna-Liisa Levonen ${ }^{1 *}$ \\ ${ }^{1}$ Department of Biotechnology and Molecular Medicine, A. I. Virtanen Institute for Molecular \\ Sciences, ${ }^{2}$ Department of Biosciences, University of Eastern Finland, FIN-70211, Kuopio, Finland, and \\ Life Sciences Research Unit, University of Luxembourg, L-1511, Luxembourg, Luxembourg. \\ * To whom correspondence should be addressed: Anna-Liisa Levonen, MD, PhD. Tel: +358 40358 \\ 9907; fax: +358 17163 751; \\ E-mail: anna-liisa.levonen@uef.fi
}

Short Title: Regulation of Bach1 via antioxidant response element

Keywords: Antioxidant, Gene expression, Gene regulation, Gene silencing, Transcription factors.

\begin{abstract}
Abbreviations: ARE, Antioxidant Response Element; BACH1, BTB and CNC homology 1; B2M, $\beta_{2-}$ microglobulin; ChIP, Chromatin Immunoprecipitation; HMOX1, Heme oxygenase-1; HUVEC, Human umbilical vein endothelial cells; Keap1, Kelch-like ECH associated protein 1; MARE, Maf recognition element; NQO1, NAD(P)H quinine oxidoreductase-1; Nrf1/NFE2L1, Nuclear Factor E2-related factor 1; Nrf2/NFE2L2, Nuclear Factor E2-related factor 2; $\mathrm{OA}-\mathrm{NO}_{2}$, nitro-oleic acid; SFN, Sulforaphane; tBHO, tert-butylhydroquinone.
\end{abstract}

\section{SYNOPSIS}

A central mechanism in cellular defence against oxidative or electrophilic stress is mediated by transcriptional induction of genes via the Antioxidant Response Element (ARE), a cis-acting sequence present in the regulatory regions of genes involved in the detoxification and elimination of reactive oxidants and electrophiles. The ARE binds different basic-region leucine zipper (bZIP) transcription factors, most notably NF-E2 related factor-2 (Nrf2) that functions as a transcriptional activator via heterodimerization with small Maf proteins. While the ARE activation by Nrf2 is relatively well understood, the mechanisms by which ARE mediated signalling is downregulated is poorly known. Transcription factor BACH1 (BTB and CNC homology 1) binds to ARE-like sequences, functioning as a transcriptional repressor in a subset of ARE-regulated genes thus antagonizing the activator function of Nrf2. Herein, we demonstrate that BACH1 itself is regulated by Nrf2 as it is induced by Nrf2 overexpression and by Nrf2 activating agents in an Nrf2-dependent manner. Furthermore, a functional ARE site was identified at +1411 from the transcription start site of transcript variant 2 of $B A C H 1$. We conclude that BACH1 is a bona fide Nrf2 target gene, and that induction of BACH1 by Nrf2 may serve as a feedback inhibitory mechanism for ARE-mediated gene regulation. 


\section{INTRODUCTION}

Cells have an inherent ability to sense and respond to environmental stress via a variety of gene regulatory pathways. A number of antioxidant and detoxification enzymes are transcriptionally induced during oxidative or electrophilic stress via the Antioxidant Response Element (ARE) [1]. ARE was initially characterized to have a cis-acting sequence with a consensus core sequence TGACnnnGC [2], and it was further characterized and extended to 5'-TMAnnRTGAYnnnGCRwwww-3' (the core ARE in bold) [3]. Subsequent analyses of the human glutamate-cysteine ligase modifier (GCLM) [4] and the mouse $\mathrm{NAD}(\mathrm{P}) \mathrm{H}$ :quinone oxidoreductase genes [5] suggest that a universally applicable ARE may not be attainable, although certain core nucleotides appear to be highly conserved [6]. Multiple basicregion leucine zipper (bZIP) transcription factors, such as those of the CNC (cap'n collar protein) family (Nrf1, Nrf2, Nrf3, and p45 NF-E2), and BTB and CNC homology proteins (BACH1 and BACH2) bind to the ARE [7]. All members from both protein families form heterodimers with small Maf proteins, which are necessary for DNA binding [7]. Transcription factors can either transactivate or repress gene expression via binding to the ARE.

Nuclear Factor E2-related factor 2 (Nrf2, NFE2L2) is one of the main ARE binding transactivators, and it regulates a wide variety of genes involved in antioxidant defense and xenobiotic metabolism, including heme oxygenase-1 (HMOX1) and NAD(P)H quinone oxidoreductase-1 (NQO1) [8]. Under basal conditions, Nrf2 is bound to its inhibitor Kelch-like ECH associated protein 1 (Keap1), where Keap1 acts as an adapter between Nrf2 and Cul3-based E3 ubiquitin ligase, facilitating proteasomal degradation of Nrf2. On exposure to oxidants or electrophiles, Nrf2 accumulates in the nucleus and drives gene expression via the ARE [9]. BACH1 is a repressor of transcription via competitive binding to the Maf recognition element (MARE), a regulatory element closely related to ARE [10]. BACH1 contains bZIP and BTB domains that are essential for DNA binding and heterodimerization. However, BACH1 lacks the transactivation domain and is therefore unable to support transcription [11]. Under unstressed conditions, BACH1 binds to its target sequence to repress gene expression. DNA binding is inhibited by heme, which interacts with several cysteine-proline (CP) motifs in the BTB domain [12]. The presence of heme also triggers the Crm1-dependent nuclear export of BACH1 [13]. It has also been proposed that oxidation of cysteine residues critical for DNA binding trigger dissociation of BACH1 from its target sequence [14]. Remarkably little is known about the target genes of BACH1 but both human and mouse HMOX1 have been unequivocally shown to be repressed by BACH1 $[15,16]$.

Transcriptional regulation of the $B A C H 1$ gene itself is poorly understood. BACH1 has three different transcript variants (1,2 and 3). Transcript variants 1 and 2 have different 5 ' untranslated regions (UTRs) but they code for the same protein, isoform A. Isoform B differs from the other two by having a 5' UTR, 3' coding region, and 3' UTR that are distinctive, resulting in a shorter protein that lacks the DNA binding properties [17]. The proposed function of the shorter isoform is to recruit the longer active isoform to the nucleus through interaction with the BTB domain. The promoter area of mouse Bach1 contains two GC boxes that bind Sp1, and these seem to be important for the regulation of basal transcription [18]. In mouse and human hepatoma cell lines, Bach1 is induced by tertbutylhydroquinone (tBHQ), which also triggers rapid nuclear export of BACH1 by phosphorylation of tyrosine 486 [19]. Human BACH1 mRNA is also induced by hypoxia through an unknown mechanism [20].

We have studied the effect of Nrf2 overexpression and nitro-oleic acid $\left(\mathrm{OA}-\mathrm{NO}_{2}\right)$-mediated activation of Nrf2 on the endothelial cell transcriptome, for the purpose of characterizing novel target genes of 
Nrf2 (Jyrkkänen HK, Kansanen E et al., unpublished data) [21]. In the course of these studies, we identified $B A C H 1$ as one of the genes potentially regulated by Nrf2. In this study we therefore investigated whether BACH1 is indeed a bona fide Nrf2 target gene. Herein, we report that adenoviral Nrf2 overexpression and Nrf2 activating compounds induce BACH1 in an Nrf2-dependent manner. Moreover, in silico analysis of the human BACH1 promoter revealed two putative ARE elements, located at +1411 and +1270 downstream of TSS of transcript variant 2 . ARE1 located at +1411 was proven to be functional using electromobility shift and promoter reporter assays, as well as by chromatin immunoprecipitation (ChIP). Our results provide a novel feedback inhibitory mechanism by which Nrf2-dependent gene expression can be inhibited via transcriptional induction of BACH1.

\section{EXPERIMENTAL PROCEDURES}

\section{Cell culture and Nrf2 inducers}

Human umbilical vein endothelial cells (HUVECs) isolated from umbilical cords obtained from the maternity ward of Kuopio University Hospital, by the approval of the Kuopio University Hospital Ethics Committee, were cultivated as described previously [22]. Human embryonic kidney 293T cells (HEK293T) were purchased from ATCC. HEK293T cells were maintained in Dulbecco's Modified Eagle's Medium (Sigma-Aldrich), supplemented with 10\% (v/v) fetal bovine serum (HyClone) and 1\% penicillin/streptomycin (Invitrogen-Gibco). Cloning and production of Nrf2 adenovirus (Ad-Nrf2) were performed as described previously [23]. Nrf2 activation was induced with sulphoraphane (SFN, SigmaAldrich), tert-butylhydroquinone (tBHQ, Sigma-Aldrich) or nitro-oleic acid $\left(\mathrm{OA}-\mathrm{NO}_{2},[24]\right)$.

\section{Cloning of plasmids}

For the luciferase assays, BACH1-ARE1, mutated elements BACH1-M1 and BACH1-M2, BACH1ARE2, wild type NQO1-ARE, and mutated NQO1-M1 were ligated into the pGL4-promoter vector (Promega) containing the minimal thymidine kinase promoter [25]. Complementary ARE containing oligonucleotides having KpnI and SacI restriction sites were synthesized (TAG Copenhagen), annealed and ligated into the vector and verified by sequencing. The sequences of the sense strands were as follows: BACH1-ARE1, 5'-CAGCTGGGGCGAATGACTCAGCAACCCAACTTTTGAGCT-3'; BACH1-M1， 5’-CAGCTGGGGCGAATAACTCAGCAACCCAACTTTTGAGCT-3'; BACH1-M2, 5'-CAGCTGGGGCGAATGACTCATTTACCCAACTTTTGAGCT-3'; $\quad$ BACH1-ARE2, 5'CAAAGGCTCCCGGTGAGTCAGCTTGGAGTAGGGTGAGCT-3’; CGATCCAGTCACAGTGACTCAGCAGAATCTGGAGCT-3'; CGATCCAGTCACAGTAACTCAGCAGAATCTGGAGCT-3’.

NQO1-ARE, $\quad 5 '-$
NQO1-M1, 5'-

\section{RNA isolation and quantitative real-time PCR}

HUVECs were transduced with Ad-Nrf2 as in [23] and harvested $48 \mathrm{~h}$ after transduction for RNA extraction. Confluent HUVECs were used for experiments with SFN, tBHQ or OA-NO $\mathrm{N}_{2}$ Total RNA was extracted with TRI-reagent (Sigma-Aldrich) and $1 \mu \mathrm{g}$ of RNA was used for the cDNA synthesis using random hexamer primers (Promega) and ReverAid M-MuLV reverse transcriptase (Fermentas Life Sciences). The relative expression levels of mRNA encoding BACH1 transcript variants 1 and 2 (Hs00230917_m1), BACH1 transcript variant 1 (Custom TaqMan Genomic assay), BACH1 transcript variant 3 (Hs01110003_s1), BACH1 all transcript variants (Hs00895421_m1) and NQO1 (Hs00168547_m1) were measured according to the manufacturer's protocol by quantitative RT-PCR (StepOnePlus ${ }^{\overline{T M}}$ Real-Time PCR systems, Applied Biosystems), using specific Assays-on-Demand 
(Applied Biosystems) target mixes. The expression levels were normalized to the level of $\beta_{2^{-}}$ microglobulin (B2M, Hs00187842_m1) (Applied Biosystems).

\section{SiRNA transfection}

HUVECs were transfected with small interfering RNA (siRNA) oligonucleotides targeting Nrf1 or Nrf2, or a non-specific siRNA control (20 nmol/L, Invitrogen) as in [26]. Forty-eight hours after transfection, cells were treated with Nrf2 activating agents for $6 \mathrm{~h}$ and mRNA levels of BACH1, NFE2L1, NFE2L2 and NQO1 were measured with quantitative real-time PCR.

\section{Luciferase reporter gene assay}

Activation of BACH-ARE or mutated elements was measured using a luciferase reporter assay. NQO1ARE and NQO1-M1 were used as controls. HEK293T cells grown in 96-well plates were transfected by using a calcium phosphate method with $100 \mathrm{ng}$ of DNA/well. BACH1-ARE or NQO1-ARE was transfected together with $\beta$-galactosidase expression vector (pCMV $\beta$; Invitrogen). In addition, pCINrf2 and p3xFLAG-Keap1 plasmids were cotransfected in some experiments. Cells were treated with $10 \mu \mathrm{M}$ SFN, $50 \mu \mathrm{M}$ tBHQ or $10 \mu \mathrm{M}$ OA-NO $24 \mathrm{~h}$ after transfection for $16 \mathrm{~h}$ and then the luciferase activity was measured using the Perkin Elmer Luciferase assay system according to the manufacturer's protocol. Luciferase activity was normalized to $\beta$-galactosidase activity as described previously [27].

\section{Electromobility shift assay (EMSA)}

For EMSA, the DNA binding region of Nrf2 (amino acids 327-605) was amplified by PCR from pCINrf2neo [28] and cloned to the KpnI and XbaI restriction sites of the pcDNA3 vector, with a Kozac sequence. MafG cDNA (German Research Center for Genome Research, Germany) was PCR amplified and cloned to the HindIII and EcoRI restriction sites of pcDNA3 vector with a Kozac sequence. The following primers were used for cloning: Nrf2 sense, 5'AATGGTACCGCCACCATGGCTTTCAACCAAAACCACCC-3'; Nrf2 antisense, 5’AAATCTAGACTAGTTTTTCTTAACATCTGGC-3'; MafG sense, 5’AATAAGCTTGCCACCATGACGACCCCCAATAAAGG-3'; and MafG antisense, 5'AAAGAATTCCTACGATCGGGCATCCGT-3'. Nrf2 and MafG proteins were generated by coupled in vitro transcription/translation using their respective pcDNA3-based cDNA expression constructs and TNT Quick Coupled Transcription/Translation kit as recommended by the supplier (Promega, Madison, WI, USA). The proteins were incubated for $10 \mathrm{~min}$ in a total volume of $20 \mu \mathrm{l}$ of binding buffer (150 mM KCl, $1 \mathrm{mM}$ dithiothreitol, $25 \mathrm{ng} / \mu \mathrm{l}$ herring sperm DNA, 5\% glycerol, $10 \mathrm{mM}$ Hepes, $\mathrm{pH}$ 7.9). [ $\left.{ }^{32} \mathrm{P}\right]$-labeled double-stranded oligonucleotides containing one copy of the respective RE were added to the mix and incubation was continued for $15 \mathrm{~min}$ at room temperature. Protein-DNA complexes were resolved by electrophoresis through $8 \%$ non-denaturing polyacrylamide gels (mono- to bisacrylamide ratio 19:1) in 0.5 x TBE (45 mM Tris, $45 \mathrm{mM}$ boric acid, $1 \mathrm{mM}$ EDTA, pH 8.3) for 100 min at $200 \mathrm{~V}$ and quantified on a FLA-3000 reader (Fuji, Tokyo, Japan) using ScienceLab99 software (Fuji). In the competition experiments, unlabeled DNA at approximately 10/50/100-fold molar excess was preincubated with protein samples for $10 \mathrm{~min}$ at $4{ }^{\circ} \mathrm{C}$ prior to the addition of the labeled probe. The reaction mixtures were incubated for an additional 15 min with the labeled probe and resolved on $8 \%$ non-denaturing polyacrylamide gels.

\section{Chromatin immunoprecipitation}

Chromatin immunoprecipitation was performed as previously described [26], with modifications. HEK293T cells were treated with $10 \mu \mathrm{M}$ SFN or DMSO as a control for $2 \mathrm{~h}$. Nuclear proteins were 
cross-linked to DNA by adding formaldehyde directly to the medium to a final concentration of $1 \%$ and incubating for $10 \mathrm{~min}$ at room temperature on a rocking platform. Cross-linking was stopped by adding glycine to a final concentration of $0.125 \mathrm{M}$ and incubating for $5 \mathrm{~min}$ at room temperature on a rocking platform. Medium was removed and the cells were washed twice with ice-cold PBS. The cells were collected and lysed with $1 \mathrm{ml}$ SDS lysis buffer (1\% SDS, $10 \mathrm{mM}$ EDTA, $50 \mathrm{mM}$ Tris-HCl, pH 8.1, protease inhibitors) and incubated at RT for $10 \mathrm{~min}$. Lysates were sonicated by a Bioruptor UCD-200 (Diagenode, Liege, Belgium) to result in DNA fragments of 200 to 1000 bp in length. Cellular debris was removed by centrifugation (14,000 rpm, $\left.15 \mathrm{~min}, 4{ }^{\circ} \mathrm{C}\right)$. After centrifugation, $20 \mu \mathrm{l}$ of each sample was separated for input control and the remaining sample was diluted 1:10 in ChIP dilution buffer (0.01\% SDS, 1.1\% Triton X-100, 1.2 mM EDTA, $167 \mathrm{mM} \mathrm{NaCl,} 16.7 \mathrm{mM}$ Tris-HCl, $\mathrm{pH}$ 8.1, protease inhibitors) and aliquoted for immunoprecipitation. $2.5 \mu \mathrm{l} \mathrm{BSA} \mathrm{(100} \mathrm{mg/ml)} \mathrm{was} \mathrm{added} \mathrm{to} \mathrm{each} \mathrm{aliquot.}$ Chromatin solutions were incubated overnight at $4{ }^{\circ} \mathrm{C}$ on a rocking platform with $3 \mu \mathrm{g}$ of a specific Nrf2 antibody (Santa Cruz Biotechnologies, sc-722), $10 \mu$ g of a specific Maf F/G/K antibody (Santa Cruz Biotechnologies, sc-22831), $10 \mu \mathrm{g}$ of an antibody against BACH1 (Santa Cruz Biotechnologies, sc-14700), or $1 \mu \mathrm{g}$ of nonspecific IgG (anti-rabbit IgG, Upstate Biotechnology, Lake Placid, NY, USA). The immunocomplexes were collected with $20 \mu \mathrm{l}$ of Magna ChIP Protein A Magnetic Beads (Millipore) for $1.5 \mathrm{~h}$ at $4{ }^{\circ} \mathrm{C}$ with rotation. The beads were separated with a magnetic rack and washed sequentially for 3 min with $700 \mu$ of the following buffers: Low Salt wash buffer $(0.1 \%$ SDS, $1 \%$ Triton X-100, 2 mM EDTA, $150 \mathrm{mM} \mathrm{NaCl,} 20 \mathrm{mM}$ Tris-HCl, pH 8.1), High Salt wash buffer $(0.1 \%$ SDS, 1\% Triton X-100, 2 mM EDTA, 500 mM NaCl, $20 \mathrm{mM}$ Tris-HCl, pH 8.1) and LiCl wash buffer (0.25 M LiCl, 1\% Nonidet P-40, 1\% sodium deoxycholate, 1 mM EDTA, $10 \mathrm{mM}$ Tris-HCl, pH 8.1). Finally, the beads were washed twice with $700 \mu \mathrm{l}$ TE buffer (1 mM EDTA, $10 \mathrm{mM}$ Tris-HCl, $\mathrm{pH}$ 8.1). The immuno-complexes were then eluted by adding $500 \mu \mathrm{l}$ of elution buffer $\left(100 \mathrm{mM} \mathrm{NaHCO}_{3}, 1 \%\right.$ SDS) and incubating for $30 \mathrm{~min}$ at RT. Proteins were digested from the eluate by adding $2 \mu \mathrm{l}$ of proteinase K (934 U/ml, Fermentas) and incubating overnight at $64{ }^{\circ} \mathrm{C}$. DNA was recovered by phenol/chloroform/isoamyl alcohol (25:24:1) extractions and precipitated with 1:10 volume of $3 \mathrm{M}$ sodium acetate, $\mathrm{pH} 5.2$, and 2 volumes of ethanol using glycogen as a carrier. Immunoprecipitated chromatin DNA was then used as a template for real-time quantitative PCR.

\section{PCR of chromatin templates}

Real-time quantitative PCR of ChIP templates was performed using chromatin region specific primers of NQO1 (primers 5'-TCCAAATCCGCAGTCACAGT-3' and 5'-TTGGCACGAAATGGAGC-3'), BACH1 (5'-TCAGCCTTCTGAAGCAACCTC-3' and 5'-TTGGAGTAGGGTGACTTTGGC-3'), HMOX1 (5'TGAGTAATCCTTTCCCGAGC-3' and 5'-GTGACTCAGCGAAAACAGACA-3') and ACTIN (5'-AACTCTCCCTCCTCCTCTTCCTC-3' and 5'-GAGCCATAAAAGGCAACTTTCGG-3'), and Maxima ${ }^{\mathrm{TM}}$ SYBR Green/ROX qPCR Master Mix in a total volume of $10 \mu \mathrm{l}$ in a LightCycler $^{\circledR} 480$ System. The PCR cycling conditions were: preincubation for $10 \mathrm{~min}$ at $95{ }^{\circ} \mathrm{C}$, 40 cycles of $20 \mathrm{~s}$ denaturation at $95{ }^{\circ} \mathrm{C}, 20 \mathrm{~s}$ annealing at $58{ }^{\circ} \mathrm{C}, 20 \mathrm{~s}$ elongation at $72{ }^{\circ} \mathrm{C}$ and a final elongation for $5 \mathrm{~min}$ at $72{ }^{\circ} \mathrm{C}$. The PCR products were also resolved on $2 \%$ agarose gels to control for correct product size. Relative association of chromatin-bound protein was calculated using the formula $2^{-(\Delta \mathrm{Ct})} * 100$, where

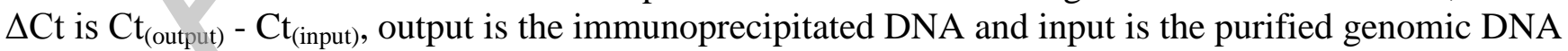
from starting material of the ChIP assay. IgG control values were subtracted from output values.

\section{Statistical analysis}


Statistical analyses were performed with GraphPad Prism Software (GraphPad Software, Inc., San Diego, CA). Statistical significance was evaluated by the unpaired t-test or one-way ANOVA using Bonferroni's post hoc comparisons. Results are expressed as mean \pm S.E.M. and differences depicted as ${ }^{*} \mathrm{p}<0.05,{ }^{* *} \mathrm{p}<0.01,{ }^{* * *} \mathrm{p}<0.001$. 


\section{RESULTS}

\section{BACH1 mRNA expression is induced by Nrf2 overexpression and Nrf2-inducing agents}

Our previous study using genome wide expression profiling indicates that BACH1 mRNA is induced by an Nrf2-activator $\mathrm{OA}-\mathrm{NO}_{2}$, suggesting that $B A C H 1$ is a transcriptional target of Nrf2 [21]. In order to verify this finding, HUVECs were transduced with Nrf2-overexpressing adenovirus with multiplicities of infection (MOI) 10 to 100, and BACH1 mRNA was measured $64 \mathrm{~h}$ after transduction. Initially, we used three different assays: an assay that measures both transcript variants 1 and 2, and assays for transcript variant 1 and transcript variant 3 . However, the expression of transcript variant 1 was not measurable (results not shown) and we therefore concluded that the assay for both variants measures transcript variant 2 in HUVECs. Both BACH1 transcript variants 2 and 3 were significantly induced by AdNrf2 with the MOI 50 and 100 (Fig. 1A). Also three structurally different Nrf2 activating agents, SFN, tBHQ and OA-NO ${ }_{2}$ increased BACH1 mRNA at $6 \mathrm{~h}$ (Fig. 1B, C and D). Using $10 \mu \mathrm{M}$ SFN as the inducer, the maximum induction was observed at $6 \mathrm{~h}$ and the mRNA levels remained elevated for $24 \mathrm{~h}$ (Fig. 1E). In addition, we examined whether the increased BACH1 mRNA expression by SFN or OA-NO 2 was a consequence of increased transcription of mRNA. For this, HUVECs were pre-treated for $1 \mathrm{~h}$ with a transcriptional inhibitor actinomycin D $(2 \mu \mathrm{g} / \mathrm{ml})$ or DMSO as a solvent control. SFN or OA-NO $\mathrm{NO}_{2}$ was then added and incubation was continued for $6 \mathrm{~h}$. SFN and OA$\mathrm{NO}_{2}$ significantly induced $B A C H 1$ mRNA in cells incubated with DMSO, whereas pre-treatment with actinomycin D abolished induction (Fig. 1F).

\section{Identification of a functional ARE binding site in the BACH1 promoter}

Considering BACH1 was effectively induced by Nrf2 overexpression and Nrf2 inducing agents, we next sought to identify whether BACH1 is a direct target of Nrf2. We therefore searched for putative ARE binding sites from the $B A C H 1$ gene in close vicinity to all three Bach1 transcript variant transcription start sites (TSSs) by in silico analysis performed with the MatInspector software (Genomatix, Germany), using a position weight matrix generated by combining 57 functional ARE sequences reported [6]. This analysis revealed two putative AREs within the first intron of the BACH1 transcript variants 1 and 2, and in the promoter region of transcript variant 3 . These two variants that are located +1411 (ARE1) and +1270 (ARE2) from the TSS of transcript variant 2 are phylogenetically conserved (Fig. 2). ARE2 is closely similar to the variant ARE described by Nioi et al. [5].

In order to examine the functionality of the two putative binding sites in the human BACH1 gene, the binding of Nrf2 to ARE1 and ARE2 was first analyzed with EMSA. An ARE from a well characterized Nrf2 target gene, NQO1, was used as a positive control. The binding of the MafG homodimer and Nrf2/MafG heterodimer to the BACH1-ARE1 was similar to that of NQO1-ARE, whereas BACH1ARE2 did not bind to the complex (Fig 3A). The specificity of Nrf2-MafG binding to the BACH1ARE1 was studied by using mutated BACH1-ARE1 oligonucleotides BACH1-M1 and BACH1-M2 (Fig. 3B). Mutations were directed to the nucleotides known to be important for Nrf2-MafG binding (Fig. 3C) [3]. The Nrf2-MafG complex failed to bind either BACH1-M1 or BACH1-M2 (Fig. 3B, lanes 8 and 12). Furthermore, binding of the Nrf2-MafG complex to the BACH1-ARE1 was inhibited with increasing concentrations of unlabeled NQO1-ARE (Fig. 3D, lanes 3-5), whereas NQO1-M1 or a nonspecific competitor (NF- $\kappa \mathrm{B}$ binding element) were not able to compete with BACH1-ARE1 binding (Fig. 3D, lanes 6-11).

To study the functionality of the two putative BACH1 AREs in a cellular context, the oligonucleotides that were used in EMSA were cloned to the luciferase vector and the effects of Nrf2 activating agents 
were tested, using NQO1-ARE as a positive control. Luciferase activity of BACH1-ARE1 and NQO1ARE was increased by all used agents (Figure 4A). The basal activity of BACH1-ARE2 was substantially lower but increased by inducing agents (Figure 4A). Of note, the basal and inducible activity of the BACH1-ARE1 was substantially lower than that of the NQO1-ARE control. Moreover, the BACH1-ARE1 harbouring the G/A mutation within the core ARE sequence (mutation M1; Fig 3C) and respective (G/T) mutation within the NQO1 ARE greatly attenuated both the basal as well as inducible ARE activity (Fig. 4A). In addition to Nrf2 inducing agents, also Nrf2 overexpression increased the activity of BACH1-ARE1 as well as NQO1-ARE as a control (Fig. 4B and C). This increase was attenuated by Keap1 cotransfection and restored by SFN and $\mathrm{OA}-\mathrm{NO}_{2}$ treatment, further affirming the notion that BACH1-ARE1 is functionally active.

To gain insight into the relevance of the findings in EMSA and reporter assays in intact cells, binding of Nrf2 and MafG with BACH1-ARE1 was studied by ChIP. The binding of Nrf2 to the BACH1ARE1 significantly increased when HEK293T cells were treated with SFN (Fig. 5A). In line with the reporter assay data, both basal as well as inducible binding of Nrf2 to NQO1-ARE relative to input control was substantially higher than to BACH1-ARE1 (Fig. 5A). ChIP using an anti-Maf antibody recognizing all three small Mafs $(\mathrm{G} / \mathrm{F} / \mathrm{K})$ indicated that small Mafs bind to both BACH1-ARE1 and NQO1-ARE with no significant increase in binding in the presence of SFN (Fig. 5B). In order to examine whether BACH1 binds to its own promoter, we performed ChIP using an antibody against BACH1 and the distal enhancer element in the HMOX1 promoter as a positive control [16, 29]. While there was significant binding to the distal enhancer region of the HMOX1 gene $(0.68 \pm 0.10 \%$ enrichment relative to input control in basal conditions, $n=3$ ), there was no detectable binding of BACH1 to the ARE of the BACH1 gene, suggesting that BACH1 does not regulate its own transcription.

\section{Induction of Bach1 expression is Nrf2 dependent}

Finally, we examined the differential role of Nrf1 and Nrf2 in the regulation of BACH1, as both factors have been shown to have a role in the regulation of ARE-dependent genes [30,31]. To this end, we used the siRNA approach (Fig. 6). The expression of NFE2L2 (Nrf2) mRNA was reduced by 83\% and NFE2L1 (Nrf1) mRNA by 89\% with respective siRNAs in basal conditions (Fig. 6A and B). Transfection with siNrf2 but not siNrf1 totally abolished both basal as well as inducible expression of NQO1 mRNA, used as a positive control (Fig. 6C). SFN treatment induced BACH1 mRNA expression by $1.8 \pm 0.2$ fold (SEM, $n=3$ ) in control siRNA transfected cells (Fig. 6D). Transfection with siNrf1 had no impact on BACH1 mRNA induction by SFN, whereas siNrf2 transfection abolished inducible expression $(86.5 \% \pm 20.4 \%$ increase by SFN treatment in siControl vs. $14.5 \% \pm 9.5 \%$ increase in siNrf2 transfected cells, mean \pm SEM, p=0.03) (Fig. 6C). Basal BACH1 expression was not significantly affected by either siNrf1 or siNrf2, suggesting that they do not play a role in the regulation of BACH1 in basal conditions.

\section{DISCUSSION}

BACH1 is a transcriptional repressor that is regulated at multiple levels. Heme binds to BACH1 and inhibits its DNA binding activity and induces nuclear export and proteasomal degradation [12, 32]. Furthermore, BACH1 protein translation is attenuated by specific microRNAs of endogenous or viral origin [33-35]. In this study, we have shown that the BACH1 gene is transcriptionally induced by Nrf2, which adds yet another layer of regulation to BACH1 expression. 
To our knowledge, this is the first study on the transcriptional regulation of the human BACH1 gene that identifies a specific transcription factor playing a regulatory role. Hypoxia has been shown to increase BACH1 mRNA with a concomitant decrease in HMOX1 expression, and it has been postulated that the human gene is regulated by hypoxia response element(s) that are not present in other species [20]. However, such elements have not been identified. Also, a recent study by Kaspar and Jaiswal that studied the nuclear export mechanisms of BACH1 in human hepatoblastoma HepG2 cells after treatment with tBHQ showed that after an initial decline, nuclear levels of BACH1 protein are restored in $4 \mathrm{~h}$ via de novo synthesis [19]. They also show that BACH1 mRNA expression is increased by tBHQ in a manner inhibitable by actinomycin $\mathrm{D}$, suggesting that increased transcription is required. Our findings are in line with this previous study and identify the mechanism by which tBHQ affects $B A C H 1$ expression.

We found a functional ARE located in the intronic region, +1411 nucleotides from the TSS of transcript variant 2. Although to our knowledge this is the first demonstration of a functional ARE element within an intron, enhancer elements are frequently found remote from the proximal 5' promoters of genes [36]. Factors that bind to these more distal regions regulate gene expression via a looping mechanism, in which they form protein-protein interactions with the general transcriptional machinery bound at the TSS. Notably, data derived from the Encyclopedia of DNA Elements (ENCODE, http://genome.ucsc.edu/ENCODE/) project reveals the presence of open chromatin using formaldehyde-assisted isolation of regulatory elements coupled with high-throughput sequencing (FAIRE-seq) HUVECs and clustering of markers of active chromatin at the vicinity of the identified ARE sequence (Supplementary Fig. 1, [37]). In addition, binding of other transcription factors was demonstrated downstream of TSS by ChIP-seq, further supporting the notion that this intron region is a highly active regulatory region.

In this study, we also examined the potential role of Nrf1 in regulating BACH1 expression. Nrf1, like other CNC-bZIP transcription factors, heterodimerizes with small Maf proteins and binds to the ARE sequence. Although Nrf1 and Nrf2 have been reported to have partially overlapping roles in the regulation of certain ARE-dependent genes such as the glutathione synthetic enzymes [38], their functions are not entirely redundant. Gene targeting of Nrf1 [39] and Nrf2 [40] in mice yields an entirely different phenotype. Moreover, gene expression profiling of hepatocyte-specific Nrf1-deficient mice revealed a distinct set of Nrf1-specific genes, such as metallothioneins-1 and -2 [30]. In our study, despite robust reduction in Nrf1 mRNA expression, neither basal nor inducible BACH1 and NQO1 mRNA expression was significantly affected by siNrf1 (Figure 6), indicating a non-redundant role of Nrf2 and Nrf1 in regulating these two genes in human endothelial cells.

Nrf2-dependent gene activation (and its inhibition by Keap1) has been a subject of intense study and it is relatively well characterized. However, little is known about the other mechanisms by which AREdependent gene expression is attenuated. BACH1 binds to the ARE-like elements within the HMOX1 promoter and represses the transcription of both human and mouse HMOX1 genes [41]. De-repression of BACH1-mediated inhibition of HO-1 transcription temporally precedes Nrf2 binding and is a prerequisite for the induction of HMOX1 by Nrf2 [16, 42]. Whether BACH1 affects the expression of other ARE-dependent genes is a matter of debate. ARE-dependent transcription of NQO1 [43] as well as ferritin and thioredoxin reductase [44] have been reported to be downregulated by BACH1. However, in studies in which BACH1 was silenced by siRNA and the gene expression was examined either by microarray analysis or qPCR, little or no effect on the expression of genes other than HMOX1 
was found [42, 45]. In the mouse Hmox1 gene all three ARE-like sequences of the distal enhancer located approximately $9 \mathrm{kB}$ upstream of TSS need to be intact for Bach1 mediated repression, and it has therefore been suggested that multiple ARE-like elements are needed for the binding of Bach1 [46]. The mechanism of this is not known, but it has been postulated that homo-oligomerization of Bach1 clustered in multiple ARE-like elements is necessary for efficient repression.

In conclusion, we have shown that $B A C H 1$ is a bona fide Nrf2 target gene regulated by an intronic ARE located at +1411 from the TSS of transcript variant 2 . We propose that after initial nuclear export and degradation of BACH1 protein, de novo synthesis via Nrf2-dependent transcriptional induction of $B A C H 1$ provides a feedback mechanism by which nuclear BACH1 levels are restored.

\section{ACKNOWLEDGEMENTS}

This study was financially supported by the Academy of Finland (ALL), the Sigrid Juselius Foundation (ALL), the Finnish Cultural Foundation (HKJ, ALL), the Finnish Foundation for Cardiovascular Research (HKJ, ALL), the Finnish Medical Foundation (HKJ), the Foundation of Aarne and Aili Turunen (HKJ), and the Finnish Cancer Organizations (ALL). We thank Dr. Sami Väisänen for the pGL4-promoter vector and Drs Bruce A. Freeman and Steven Woodcock for the generous gift of nitro-oleic acid.

\section{REFERENCES}

1 Nguyen, T., Sherratt, P. J. and Pickett, C. B. (2003) Regulatory mechanisms controlling gene expression mediated by the antioxidant response element. Annu. Rev. Pharmacol. Toxicol. 43, 233260. doi:10.1146/annurev.pharmtox.43.100901.140229

2 Rushmore, T. H., Morton, M. R. and Pickett, C. B. (1991) The antioxidant responsive element. activation by oxidative stress and identification of the DNA consensus sequence required for functional activity. J. Biol. Chem. 266, 11632-11639

3 Wasserman, W. W. and Fahl, W. E. (1997) Functional antioxidant responsive elements. Proc. Natl. Acad. Sci. U. S. A. 94, 5361-5366

4 Erickson, A. M., Nevarea, Z., Gipp, J. J. and Mulcahy, R. T. (2002) Identification of a variant antioxidant response element in the promoter of the human glutamate-cysteine ligase modifier subunit gene. revision of the ARE consensus sequence. J. Biol. Chem. 277, 30730-30737. doi:10.1074/jbc.M205225200

5 Nioi, P., McMahon, M., Itoh, K., Yamamoto, M. and Hayes, J. D. (2003) Identification of a novel Nrf2-regulated antioxidant response element (ARE) in the mouse NAD(P)H:Quinone oxidoreductase 1 gene: Reassessment of the ARE consensus sequence. Biochem. J. 374, 337-348. doi:10.1042/BJ20030754

6 Wang, X., Tomso, D. J., Chorley, B. N., Cho, H. Y., Cheung, V. G., Kleeberger, S. R. and Bell, D. A. (2007) Identification of polymorphic antioxidant response elements in the human genome. Hum. Mol. Genet. 16, 1188-1200. doi:10.1093/hmg/ddm066 
7 Kensler, T. W., Wakabayashi, N. and Biswal, S. (2007) Cell survival responses to environmental stresses via the Keap1-Nrf2-ARE pathway. Annu. Rev. Pharmacol. Toxicol. 47, 89116. doi:10.1146/annurev.pharmtox.46.120604.141046

8 Itoh, K., Tong, K. I. and Yamamoto, M. (2004) Molecular mechanism activating Nrf2-Keap1 pathway in regulation of adaptive response to electrophiles. Free Radic. Biol. Med. 36, 1208-1213. doi:10.1016/j.freeradbiomed.2004.02.075

9 Zhang, D. D. (2006) Mechanistic studies of the Nrf2-Keap1 signaling pathway. Drug Metab. Rev. 38, 769-789. doi:10.1080/03602530600971974

10 Igarashi, K. and Sun, J. (2006) The heme-Bach1 pathway in the regulation of oxidative stress response and erythroid differentiation. Antioxid. Redox Signal. 8, 107-118. doi:10.1089/ars.2006.8.107

11 Igarashi, K., Hoshino, H., Muto, A., Suwabe, N., Nishikawa, S., Nakauchi, H. and Yamamoto, M. (1998) Multivalent DNA binding complex generated by small maf and Bach1 as a possible biochemical basis for beta-globin locus control region complex. J. Biol. Chem. 273, 11783-11790

12 Ogawa, K., Sun, J., Taketani, S., Nakajima, O., Nishitani, C., Sassa, S., Hayashi, N., Yamamoto, M., Shibahara, S., Fujita, H. and Igarashi, K. (2001) Heme mediates derepression of maf recognition element through direct binding to transcription repressor Bach1. EMBO J. 20, 2835-2843. doi:10.1093/emboj/20.11.2835

13 Suzuki, H., Tashiro, S., Hira, S., Sun, J., Yamazaki, C., Zenke, Y., Ikeda-Saito, M., Yoshida, M. and Igarashi, K. (2004) Heme regulates gene expression by triggering Crm1-dependent nuclear export of Bach1. EMBO J. 23, 2544-2553. doi:10.1038/sj.emboj.7600248

14 Ishikawa, M., Numazawa, S. and Yoshida, T. (2005) Redox regulation of the transcriptional repressor Bach1. Free Radic. Biol. Med. 38, 1344-1352. doi:10.1016/j.freeradbiomed.2005.01.021

15 Sun, J., Hoshino, H., Takaku, K., Nakajima, O., Muto, A., Suzuki, H., Tashiro, S., Takahashi, S., Shibahara, S., Alam, J., Taketo, M. M., Yamamoto, M. and Igarashi, K. (2002) Hemoprotein Bach1 regulates enhancer availability of heme oxygenase-1 gene. EMBO J. 21, 5216-5224

16 Reichard, J. F., Motz, G. T. and Puga, A. (2007) Heme oxygenase-1 induction by NRF2 requires inactivation of the transcriptional repressor BACH1. Nucleic Acids Res. 35, 7074-7086. doi:10.1093/nar/gkm638

17 Kanezaki, R., Toki, T., Yokoyama, M., Yomogida, K., Sugiyama, K., Yamamoto, M., Igarashi, K. and Ito, E. (2001) Transcription factor BACH1 is recruited to the nucleus by its novel alternative spliced isoform. J. Biol. Chem. 276, 7278-7284. doi:10.1074/jbc.M004227200

18 Sun, J., Muto, A., Hoshino, H., Kobayashi, A., Nishimura, S., Yamamoto, M., Hayashi, N., Ito, E. and Igarashi, K. (2001) The promoter of mouse transcription repressor bach1 is regulated by Sp1 and trans-activated by Bach1. J. Biochem. 130, 385-392 
19 Kaspar, J. W. and Jaiswal, A. K. (2010) Antioxidant-induced phosphorylation of tyrosine 486 leads to rapid nuclear export of Bach1 that allows Nrf2 to bind to the antioxidant response element and activate defensive gene expression. J. Biol. Chem. 285, 153-162. doi:10.1074/jbc.M109.040022

20 Kitamuro, T., Takahashi, K., Ogawa, K., Udono-Fujimori, R., Takeda, K., Furuyama, K., Nakayama, M., Sun, J., Fujita, H., Hida, W., Hattori, T., Shirato, K., Igarashi, K. and Shibahara, S. (2003) Bach1 functions as a hypoxia-inducible repressor for the heme oxygenase-1 gene in human cells. J. Biol. Chem. 278, 9125-9133. doi:10.1074/jbc.M209939200

21 Kansanen, E., Jyrkkanen, H. K., Volger, O. L., Leinonen, H., Kivela, A. M., Hakkinen, S. K., Woodcock, S. R., Schopfer, F. J., Horrevoets, A. J., Yla-Herttuala, S., Freeman, B. A. and Levonen, A. L. (2009) Nrf2-dependent and -independent responses to nitro-fatty acids in human endothelial cells: Identification of heat shock response as the major pathway activated by nitro-oleic acid. J. Biol. Chem. 284, 33233-33241. doi:10.1074/jbc.M109.064873

22 Levonen, A. L., Dickinson, D. A., Moellering, D. R., Mulcahy, R. T., Forman, H. J. and Darley-Usmar, V. M. (2001) Biphasic effects of 15-deoxy-delta(12,14)-prostaglandin J(2) on glutathione induction and apoptosis in human endothelial cells. Arterioscler. Thromb. Vasc. Biol. 21, 1846-1851

23 Levonen, A. L., Inkala, M., Heikura, T., Jauhiainen, S., Jyłkkanen, H. K., Kansanen, E., Maatta, K., Romppanen, E., Turunen, P., Rutanen, J. and Yla-Herttuala, S. (2007) Nrf2 gene transfer induces antioxidant enzymes and suppresses smooth muscle cell growth in vitro and reduces oxidative stress in rabbit aorta in vivo. Arterioscler. Thromb. Vasc. Biol. 27, 741-747. doi:10.1161/01.ATV.0000258868.80079.4d

24 Woodcock, S. R., Marwitz, A. J., Bruno, P. and Branchaud, B. P. (2006) Synthesis of nitrolipids. all four possible diastereomers of nitrooleic acids: (E)- and (Z)-, 9- and 10-nitro-octadec9-enoic acids. Org. Lett. 8, 3931-3934. doi:10.1021/ol0613463

25 Turunen, M. M., Dunlop, T. W., Carlberg, C. and Vaisanen, S. (2007) Selective use of multiple vitamin D response elements underlies the 1 alpha,25-dihydroxyvitamin D3-mediated negative regulation of the human CYP27B1 gene. Nucleic Acids Res. 35, 2734-2747. doi:10.1093/nar/gkm179

26 Jyrkkanen, H. K., Kansanen, E., Inkala, M., Kivela, A. M., Hurttila, H., Heinonen, S. E., Goldsteins, G., Jauhiainen, S., Tiainen, S., Makkonen, H., Oskolkova, O., Afonyushkin, T., Koistinaho, J., Yamamoto, M., Bochkov, V. N., Yla-Herttuala, S. and Levonen, A. L. (2008) Nrf2 regulates antioxidant gene expression evoked by oxidized phospholipids in endothelial cells and murine arteries in vivo. Circ. Res. 103, e1-9. doi:10.1161/CIRCRESAHA.108.176883

27 Levonen, A. L., Landar, A., Ramachandran, A., Ceaser, E. K., Dickinson, D. A., Zanoni, G., Morrow, J. D. and Darley-Usmar, V. M. (2004) Cellular mechanisms of redox cell signalling: Role of cysteine modification in controlling antioxidant defences in response to electrophilic lipid oxidation products. Biochem. J. 378, 373-382. doi:10.1042/BJ20031049 
28 Wild, A. C., Moinova, H. R. and Mulcahy, R. T. (1999) Regulation of gammaglutamylcysteine synthetase subunit gene expression by the transcription factor Nrf2. J. Biol. Chem. 274, 33627-33636

29 Kansanen, E., Bonacci, G., Schopfer, F. J., Kuosmanen, S. M., Tong, K. I., Leinonen, H., Woodcock, S. R., Yamamoto, M., Carlberg, C., Yla-Herttuala, S., Freeman, B. A. and Levonen, A. L. (2011) Electrophilic nitro-fatty acids activate NRF2 by a KEAP1 cysteine 151-independent mechanism. J. Biol. Chem. 286, 14019-14027. doi:10.1074/jbc.M110.190710

30 Ohtsuji, M., Katsuoka, F., Kobayashi, A., Aburatani, H., Hayes, J. D. and Yamamoto, M. (2008) Nrf1 and Nrf2 play distinct roles in activation of antioxidant response element-dependent genes. J. Biol. Chem. 283, 33554-33562. doi:10.1074/jbc.M804597200

31 Biswas, M. and Chan, J. Y. (2010) Role of Nrf1 in antioxidant response element-mediated gene expression and beyond. Toxicol. Appl. Pharmacol. 244, 16-20. doi:10.1016/j.taap.2009.07.034

32 Zenke-Kawasaki, Y., Dohi, Y., Katoh, Y., Ikura, T., Ikura, M., Asahara, T., Tokunaga, F., Iwai, K. and Igarashi, K. (2007) Heme induces ubiquitination and degradation of the transcription factor Bach1. Mol. Cell. Biol. 27, 6962-6971. doi:10.1128/MCB.02415-06

33 Hou, W., Tian, Q., Zheng, J. and Bonkovsky, H. L. (2010) MicroRNA-196 represses Bach1 protein and hepatitis $\mathrm{C}$ virus gene expression in human hepatoma cells expressing hepatitis $\mathrm{C}$ viral proteins. Hepatology. 51, 1494-1504. doi:10.1002/hep.23401

34 Gottwein, E., Mukherjee, N., Sachse, C., Frenzel, C., Majoros, W. H., Chi, J. T., Braich, R., Manoharan, M., Soutschek, J., Ohler, U. and Cullen, B. R. (2007) A viral microRNA functions as an orthologue of cellular miR-155. Nature. 450, 1096-1099. doi:10.1038/nature05992

35 Shan, Y., Zheng, J., Lambrecht, R. W. and Bonkovsky, H. L. (2007) Reciprocal effects of micro-RNA-122 on expression of heme oxygenase-1 and hepatitis $\mathrm{C}$ virus genes in human hepatocytes. Gastroenterology. 133, 1166-1174. doi:10.1053/j.gastro.2007.08.002

36 Farnham, P. J. (2009) Insights from genomic profiling of transcription factors. Nat. Rev. Genet. 10, 605-616. doi:10.1038/nrg2636

37 ENCODE Project Consortium, Birney, E. et. al. (2007) Identification and analysis of functional elements in $1 \%$ of the human genome by the ENCODE pilot project. Nature. 447, 799816. doi:10.1038/nature05874

38 Kwong, M., Kan, Y. W. and Chan, J. Y. (1999) The CNC basic leucine zipper factor, Nrf1, is essential for cell survival in response to oxidative stress-inducing agents. role for Nrf1 in gammagcs(l) and gss expression in mouse fibroblasts. J. Biol. Chem. 274, 37491-37498

39 Chan, J. Y., Kwong, M., Lu, R., Chang, J., Wang, B., Yen, T. S. and Kan, Y. W. (1998) Targeted disruption of the ubiquitous CNC-bZIP transcription factor, nrf-1, results in anemia and embryonic lethality in mice. EMBO J. 17, 1779-1787. doi:10.1093/emboj/17.6.1779 
40 Itoh, K., Chiba, T., Takahashi, S., Ishii, T., Igarashi, K., Katoh, Y., Oyake, T., Hayashi, N., Satoh, K., Hatayama, I., Yamamoto, M. and Nabeshima, Y. (1997) An Nrf2/small maf heterodimer mediates the induction of phase II detoxifying enzyme genes through antioxidant response elements. Biochem. Biophys. Res. Commun. 236, 313-322

41 Ryter, S. W., Alam, J. and Choi, A. M. (2006) Heme oxygenase-1/carbon monoxide: From basic science to therapeutic applications. Physiol. Rev. 86, 583-650. doi:10.1152/physrev.00011.2005

42 Reichard, J. F., Sartor, M. A. and Puga, A. (2008) BACH1 is a specific repressor of HMOX1 that is inactivated by arsenite. J. Biol. Chem. 283, 22363-22370. doi:10.1074/jbc.M801784200

43 Dhakshinamoorthy, S., Jain, A. K., Bloom, D. A. and Jaiswal, A. K. (2005) Bach1 competes with Nrf2 leading to negative regulation of the antioxidant response element (ARE)-mediated NAD(P)H:Quinone oxidoreductase 1 gene expression and induction in response to antioxidants. J. Biol. Chem. 280, 16891-16900. doi:10.1074/jbc.M500166200

44 Hintze, K. J., Katoh, Y., Igarashi, K. and Theil, E. C. (2007) Bach1 repression of ferritin and thioredoxin reductase 1 is heme-sensitive in cells and in vitro and coordinates expression with heme oxygenase1, beta-globin, and NADP(H) quinone (oxido) reductase1. J. Biol. Chem. 282, 3436534371. doi:10.1074/jbc.M700254200

45 MacLeod, A. K., McMahon, M., Plummer, S. M., Higgins, L. G., Penning, T. M., Igarashi, K. and Hayes, J. D. (2009) Characterization of the cancer chemopreventive NRF2-dependent gene battery in human keratinocytes: Demonstration that the KEAP1-NRF2 pathway, and not the BACH1-NRF2 pathway, controls cytoprotection against electrophiles as well as redox-cycling compounds. Carcinogenesis. 30, 1571-1580. doi:10.1093/carcin/bgp176

46 Dohi, Y., Alam, J., Yoshizumi, M., Sun, J. and Igarashi, K. (2006) Heme oxygenase-1 gene enhancer manifests silencing activity in a chromatin environment prior to oxidative stress. Antioxid. Redox Signal. 8, 60-67. doi:10.1089/ars.2006.8.60

\section{FIGURE LEGENDS}

Figure 1. Adenoviral Nrf2 over-expression and Nrf2 activation induce BACH1 mRNA.

(A-E), HUVECs were transduced with Nrf2 over-expressing adenovirus using multiplicities of infection 10 to 100 for $64 \mathrm{~h}$ (A), or were treated with SFN (B), tBHQ (C) or OA-NO $(\mathrm{D})$ for $6 \mathrm{~h}$ or with $10 \mu \mathrm{M}$ SFN for 0-24 h (E). (F) After $1 \mathrm{~h}$ pre-treatment with actinomycin $\mathrm{D}(2 \mu \mathrm{g} / \mathrm{ml})$ or DMSO, HUVECs were treated with $10 \mu \mathrm{M}$ SFN or $5 \mu \mathrm{M}$ OA-NO $\mathrm{NO}_{2}$ for $6 \mathrm{~h}$. BACH1 mRNA expression was measured with quantitative real-time PCR and normalized to B2M expression. Values are presented as mean \pm S.E.M. $(n=3) .{ }^{*} \mathrm{p}<0.05,{ }^{* *} \mathrm{p}<0.01,{ }^{* * *} \mathrm{p}<0.001$ vs. control. ${ }^{\circ} \mathrm{p}<0.05$, ${ }_{\mathrm{p}}^{\mathrm{p}}<0.001$, DMSO vs. actinomycin D. 
Figure 2. BACH1 gene structure, sequence and promoter alignment.

(A) $B A C H 1$ gene structure. $B A C H 1$ has three transcript variants (1-3). Transcript variants 1 and 2 have the same exon structure and code for the same protein. Transcript variant 3 lacks the first exon and has two more exons at the 3' end in comparison to the others. Location of the two putative ARE elements in the BACH1 gene are presented. (B) Sequences of the putative BACH1 ARE elements were aligned with the ARE consensus sequence [3] and the NQO1-ARE sequence. The BACH1 ARE1 is in reverse orientation. (C) Human $B A C H 1$ promoter sequence aligned with the mouse sequence.

Figure 3. Nrf2-MafG protein complex binds to the human BACH1 ARE.

(A) Labeled oligonucleotides were incubated with in vitro translated MafG (lanes 2, 6, and 10), Nrf2 (lanes 3, 7, and 11) or both $(4,6,12)$. (B) ${ }^{32}$ P-labeled BACH1-ARE1 or ARE1 mutations M1 or M2 (sequences in panel C) were incubated with in vitro translated MafG (lanes 2, 6, and 10), Nrf2 (lanes 3, 7, and 11), or both (lanes 4, 8, and 12). (D) ${ }^{32}$ P-labeled BACH1-ARE was incubated with increasing concentrations of unlabeled NQO1-ARE (lanes 3-5), NQO1-M1 (lanes 6-8), or NF-кB element (lanes 9-11).

Figure 4. Nrf2 cotransfection or Nrf2 activators increase human BACH1-ARE activity. (A) HEK293T cells were transfected with wild type or mutated BACH1-ARE1 or BACH1-ARE2 cloned into pGL4-promoter vectors. The cells were treated with SFN, tBHQ or OA-NO 24 h after transfection, and luciferase activity was measured 16 h thereafter. NQO1-ARE and NQO1-M1 were used as controls. (B) and (C): HEK293T cells were transfected with BACH1-ARE1 (B) or NQO1ARE (C) constructs with Nrf2- and Keap1- expressing plasmids. Twenty-four hours after transfection, cells were treated with Nrf2 activating agents for $16 \mathrm{~h}$ and luciferase activities were measured. An empty pGL4-promoter vector was used as a control in all experiments. Luciferase activities were normalized to $\beta$-galactosidase activity. Values are shown relative to control and presented as mean \pm S.E.M., $(n=5-6),{ }^{*} \mathrm{p}<0.05$ vs, relative control.

Figure 5. Binding of Nrf2 and MafG to the ARE of human BACH1 and NQO1.

(A, B) HEK293T cells were treated with $10 \mu \mathrm{M}$ SFN for $2 \mathrm{~h}$ and ChIP performed using anti-Nrf2 (A) and anti-MafF/G/K antibodies (B). Real-time quantitative PCR was performed using primers specific for an ARE of BACH1, NQO1, and ACTIN genes. Binding is depicted as \% of input values from three independent experiments. Values are presented as mean \pm S.E.M. $(\mathrm{n}=3) .{ }^{*} \mathrm{p}<0.05$ vs. control treatment.

Figure 6. SFN induced BACH1 expression is Nrf2 dependent.

HUVECs were transfected with control, Nrf1 or Nrf2 siRNA. Forty-eight hours after transfection, cells were treated with SFN for $6 \mathrm{~h}$ and mRNA levels of NFE2L2 (A), NFE2L1 (B) BACH1 (C), and NQO1 (D) genes were measured with quantitative real-time PCR. The results are normalized to B2M and values are presented as mean \pm SEM $(n=3)$. (A) and (B): The results are depicted as fold change vs. control siRNA transfected, untreated cells $\# \mathrm{p}<0.05$, or vs. control siRNA transfected, SFN treated cells, ap $<0.05$. (C) and (D): The results are depicted as fold change untreated cells vs. treated cells, ${ }^{*} \mathrm{p}<0.05$. 

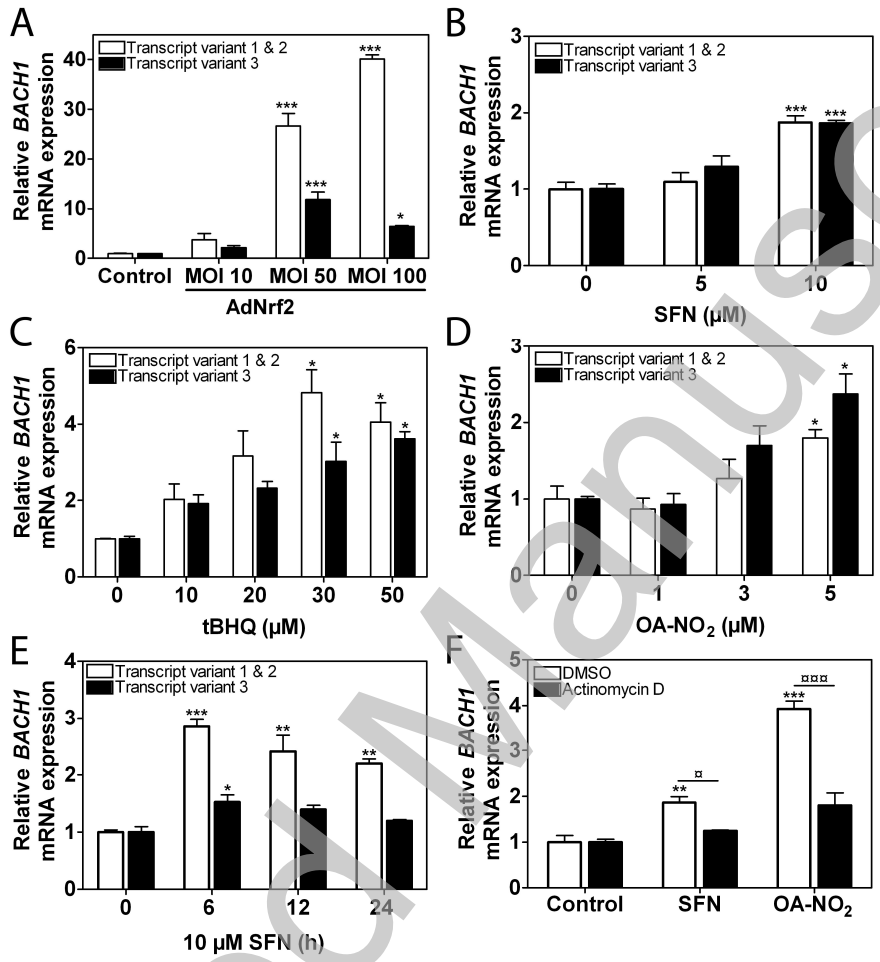

Figure 1 Jyrkkänen HK et al 2011 
A

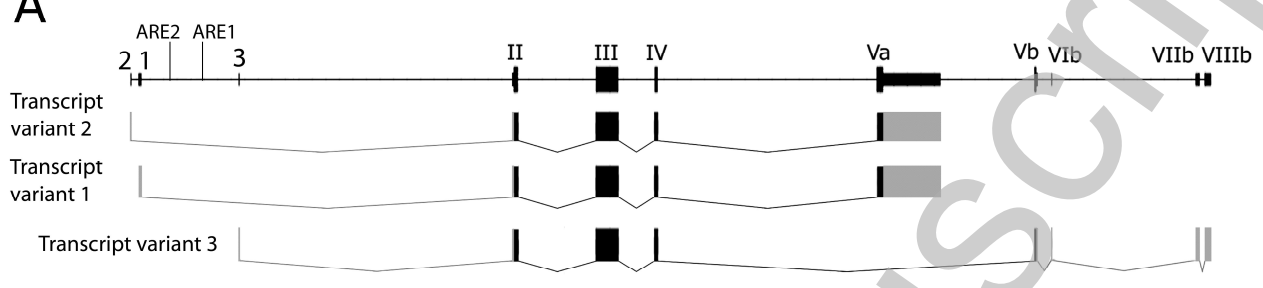

B

$\begin{array}{ll}\text { ARE Consensus } & \text { TMANNRTAYNNNGCRWWWW } \\ \text { NQO1-ARE } & \text { TCACAGTGACTCAGCAGAATC } \\ \text { BACH1-ARE1 } & \text { GGCGAATGACTCAGCAACCCA } \\ \text { BACH1-ARE2 } & \text { TCCCGGTGAGTCAGCTTGGAG }\end{array}$

$+1220 \quad$ ARE 2

Human CACTTGCCACTTCTGCCTTTGGACATCTTGAAGAAGCTTA-GGGAAGAAAGGCTCCCGGTGAGTCAGCTTGGAGTAGGGTGACTTTGG

Mouse CGCTTGCCACTTCTGGCTTTGGACATCGTAAGGGAGTCTAGAGGAAAAAAGCTCCTGGTGAGTCATCITGGAGTTGGGTAGCTTTGG

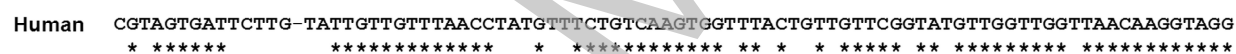

Mouse AGGAGTGATCGCCTC--TTGTTGTTTAACCGTCGGATCTGTCAAGTGGCTTCCCTTTGTTCGCTACGTTGGTTGGATAACAAGGTAGG ARE 1

Human ATGACTCTCTAAAAAG--TTGGTTGCT GAGTCATTCGCCCAGCTTGAGAGTAATTAGAAACAGCTGGTGCAAATACTCTCATCAC

Mouse GTGACTCTTPAAAGATTTGTGTTGCT GAGTCATTCACCCCAGACTGAGAGTAATTAGGAACAGCTGGTGCAGATACGCGCATCAC

Human +1543

Mouse ATGCCTCCACTATAAAAGGCTGAGCTGCACACACGAGGGGTGGCTTCAGGAGGCCGGAGGGGG

Figure 2 Jyrkkänen HK et al 2011 


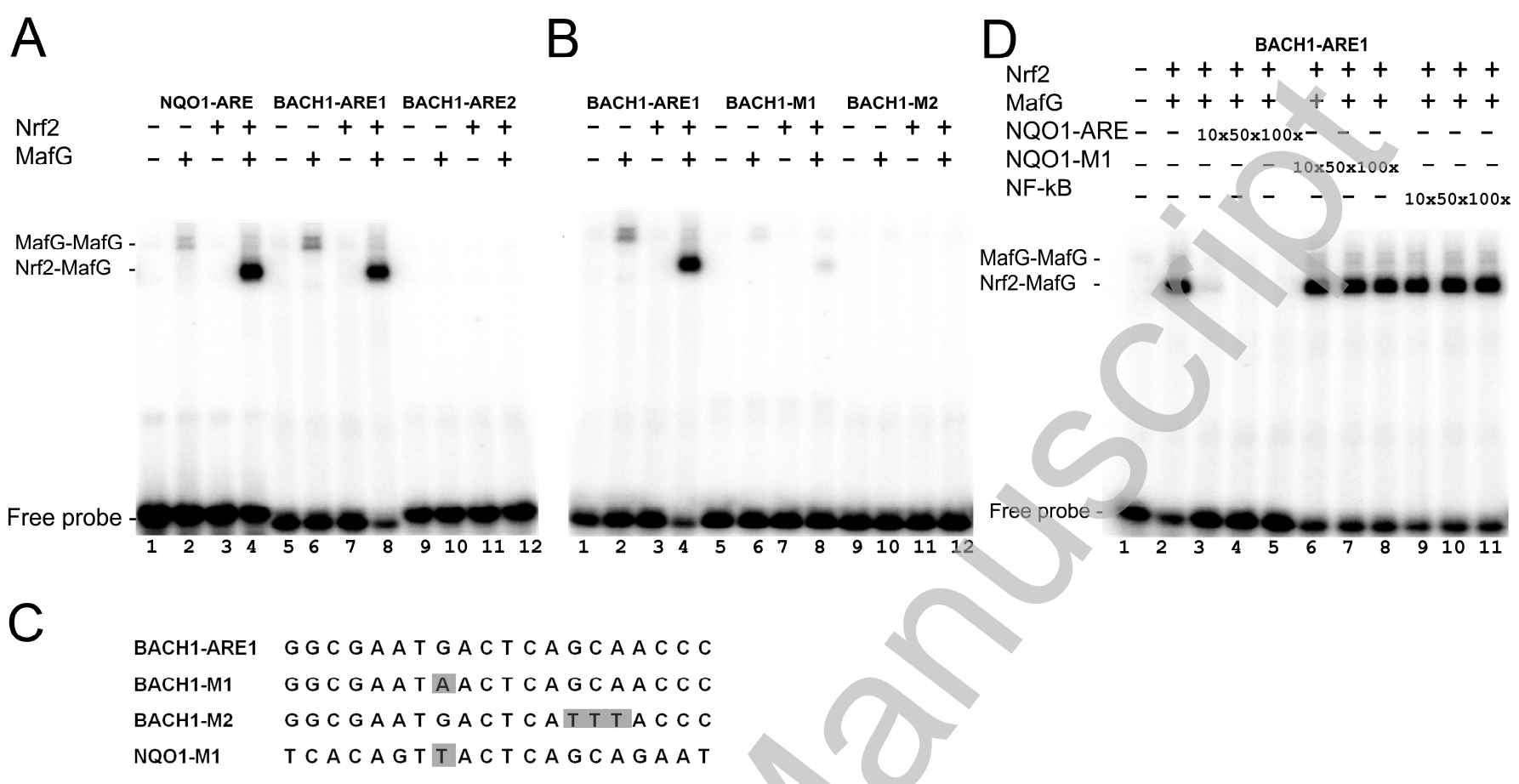

Figure 3 Jyrkkänen HK et al 2011

Licenced copy. Copying is not permitted, except with prior permission and as allowed by law. 


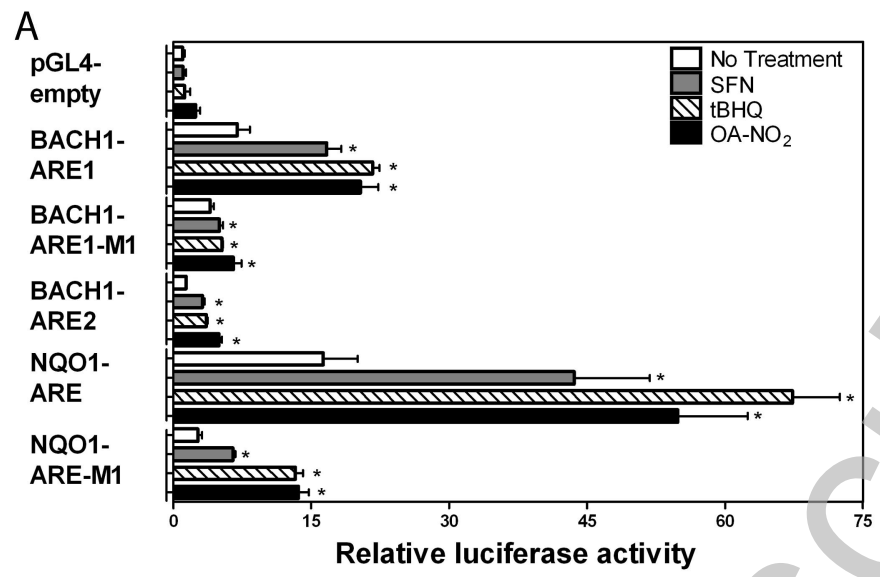

B
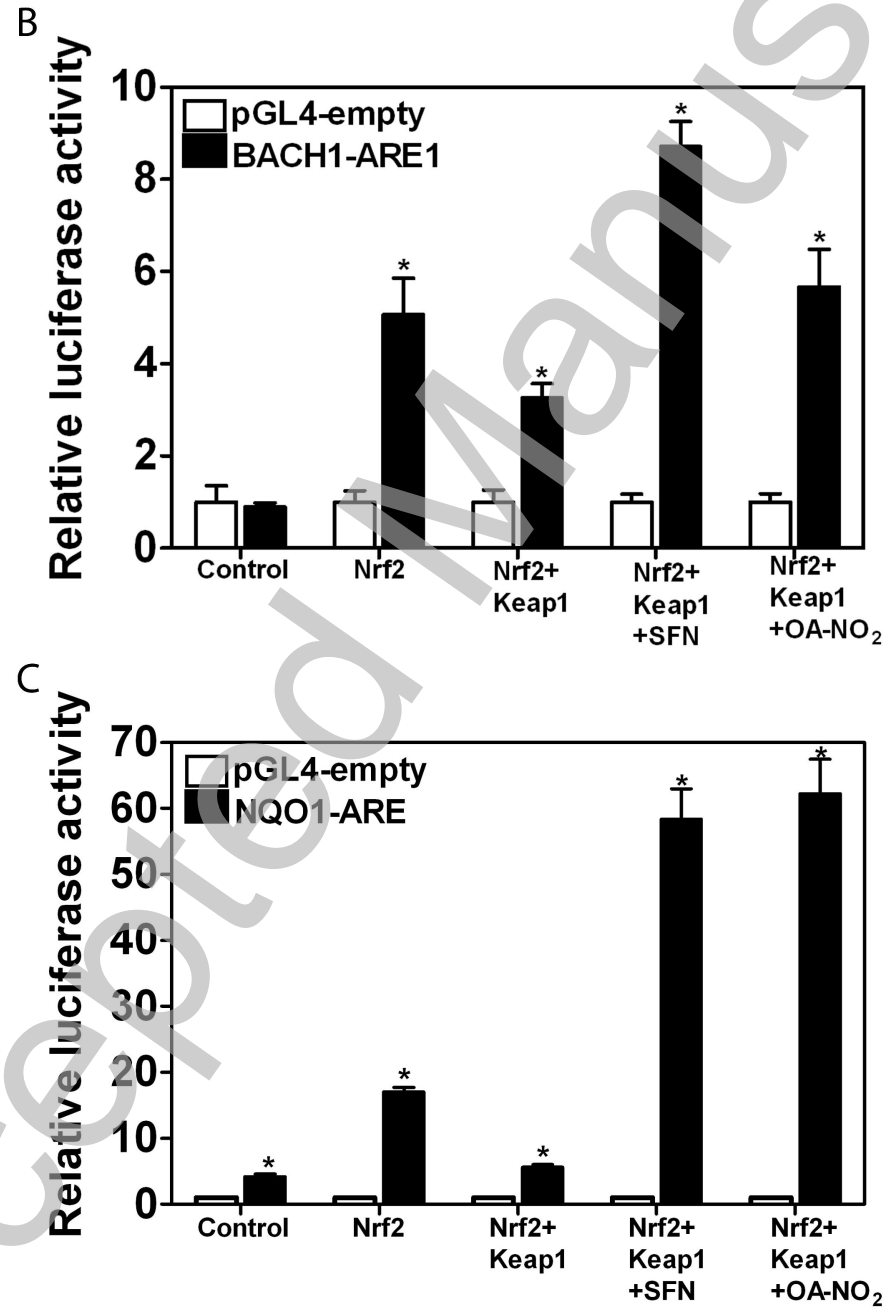

Figure 4 Jyrkkänen HK et al 2011 

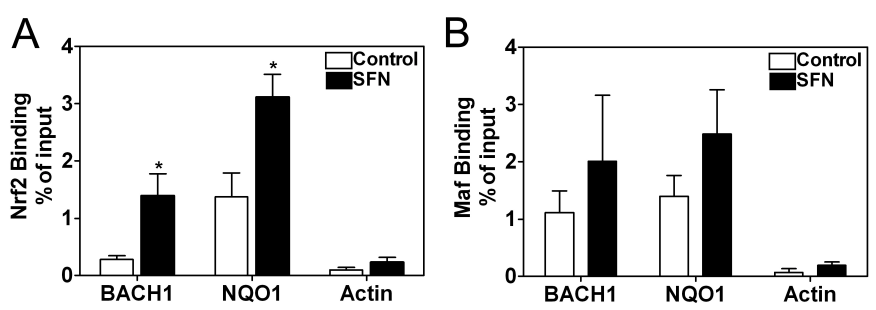

Figure 5 Jyrkkänen HK et al 2011

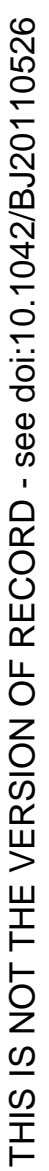

Licenced copy. Copying is not permitted, except with prior permission and as allowed by law. 

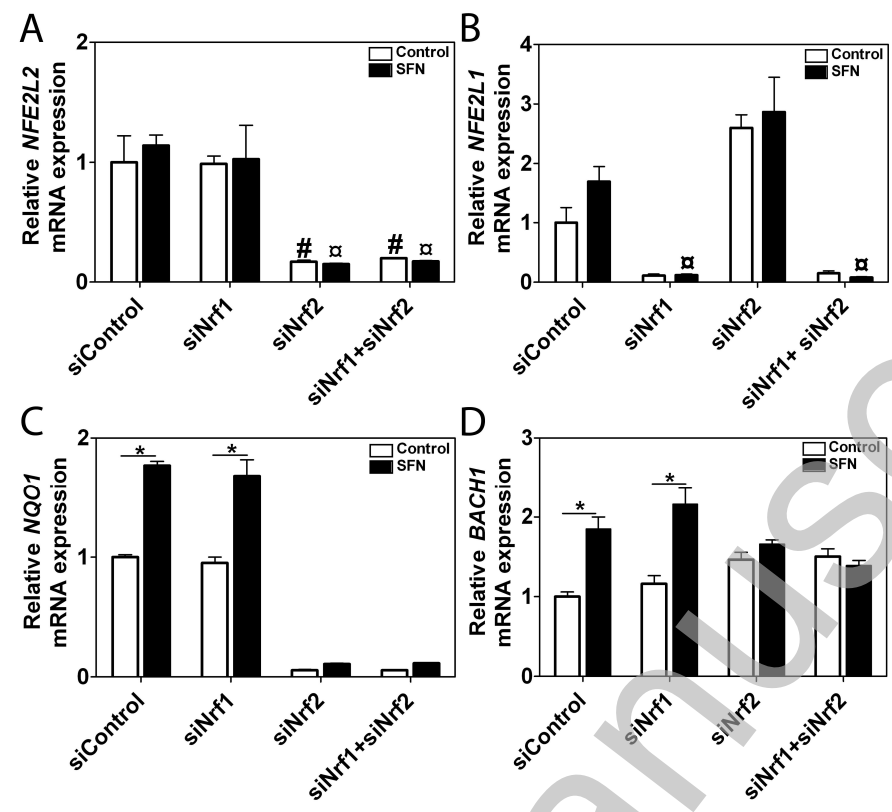

Figure 6 Jyrkkänen HK et al 2011 